\title{
Re: Enhancing Informed Consent for Research and Treatment ${ }^{\text {负 }}$
}

The lead article in the June issue of Neuropsychopharmacology is an important contribution entitled "Enhancing informed consent for research and treatment." Drs. Dunn and Jeste (2001) did an excellent job, but their extensive review apparently missed one of the earliest papers in the literature dealing with a strategy to improve informed consent in psychiatric patients. This is the simple technique developed during the 1970s in our Center for Studies of Addiction. It consists of giving a quiz on the protocol after explanation to the patient and not allowing the volunteer to sign until they have taken the quiz and corrected any wrong answers. We published a paper on this in 1979 (Grabowski et al. 1979) and have used the technique successfully in our clinical research for about a quarter of a century. On at least one occasion it received external verification by a National Academy of Science field survey of the Veterans Administration. The interviewers found that research subjects throughout the VA had an average of $27 \%$ "unaware of the general nature of the research" while none of those interviewed from our Center were found to be "unaware."
In summary, we salute the authors for an excellent review and for calling attention to ways of enhancing informed consent. We only wish to point out that the consent form quiz as we have used it for over a quarter of a century works very well. In recent years it has been adopted by all investigators at our medical center and the quiz as well as the consent form are reviewed by our hospital's IRB.

Charles P. O'Brien, M.D, Ph.D VA Medical Center Philadelphia, PA

\section{REFERENCES}

Grabowski J, O'Brien CP, Mintz J (1979): Increasing the likelihood that consent is informed. J of Exp Analysis of Behav 24:283-284

Jeste D, Dunn LB (2001): Enhancing Informed Consent for Research and Treatment. Neuropsychopharmacology 24:595-607

Refers to PII S0893-133X(00)00218-9 